\title{
Role of proline and pyrroline-5-carboxylate metabolism in plant defense against invading pathogens
}

\author{
Aarzoo Qamar', Kirankumar S. Mysore ${ }^{2}$ and Muthappa Senthil-Kumar ${ }^{1 *}$ \\ ${ }^{1}$ National Institute of Plant Genome Research, New Delhi, India, ${ }^{2}$ Plant Biology Division, The Samuel Roberts Noble \\ Foundation, Ardmore, OK, USA
}

OPEN ACCESS

Edited by:

Dietmar Funck,

University of Konstanz, Germany

Reviewed by:

Paul E. Verslues, Academia Sinica, Taiwan

Yasuhiro Ishiga,

University of Tsukuba, Japan

*Correspondence: Muthappa Senthil-Kumar, National Institute of Plant Genome Research, Aruna Asaf Ali Marg, P. O. Box 10531, New Delhi 110067, India skmuthappa@nipgr.ac.in

Specialty section: This article was submitted to Plant Physiology, a section of the journal Frontiers in Plant Science

Received: 29 April 2015

Accepted: 22 June 2015

Published: 06 July 2015

Citation:

Qamar A, Mysore KS and Senthil-Kumar M (2015) Role of proline and pyrroline-5-carboxylate metabolism in plant defense against

invading pathogens.

Front. Plant Sci. 6:503.

doi: 10.3389/fpls.2015.00503
Pyrroline-5-carboxylate (P5C) is an intermediate product of both proline biosynthesis and catabolism. Recent evidences indicate that proline-P5C metabolism is tightly regulated in plants, especially during pathogen infection and abiotic stress. However, role of P5C and its metabolism in plants has not yet been fully understood. Studies indicate that P5C synthesized in mitochondria has a role in both resistance $(R)$-gene-mediated and non-host resistance against invading pathogens. Proline dehydrogenase and delta-ornithine amino transferase-encoding genes, both involved in P5C synthesis in mitochondria are implicated in defense response of Nicotiana benthamiana and Arabidopsis thaliana against bacterial pathogens. Such defense response is proposed to involve salicylic acid-dependent pathway, reactive oxygen species (ROS) and hypersensitive response (HR)-associated cell death. Recently HR, a form of programmed cell death (PCD), has been proposed to be induced by changes in mitochondrial P5C synthesis or the increase in P5C levels per se in plants inoculated with either a host pathogen carrying suitable avirulent (Avr) gene or a non-host pathogen. Consistently, A. thaliana mutant plants deficient in P5C catabolism showed HR like cell death when grown in external P5C or proline supplemented medium. Similarly, yeast and plant cells under oxidative stress were shown to increase ROS production and PCD due to increase in P5C levels. Similar mechanism has also been reported as one of the triggers for apoptosis in mammalian cells. This review critically analyzes results from various studies and enumerates the pathways for regulation of P5C levels in the plant cell, especially in mitochondria, during pathogen infection. Further, mechanisms regulating P5C- mediated defense responses, namely $\mathrm{HR}$ are outlined. This review also provides new insights into the differential role of proline-P5C metabolism in plants exposed to pathogen infection.

Keywords: P5C, proline, ROS, oxidative burst, hypersensitive response, plant defense, non-host resistance 


\section{Introduction}

Plant defense against invading pathogen involves complex responses that culminate either in plant susceptibility or resistance. Virulent pathogen colonizes on host plant by surpassing the plant resistance mechanism. Most virulent pathogens have capability to manipulate host gene expression patterns to directly benefit their fitness and cause susceptibility of host plant. However, avirulent pathogen infection provokes $R$-gene mediated resistance owing to recognition of avirulent factor by resistance protein (R-protein). This recognition leads to hypersensitive response (HR)-cell death or other defense responses. Further, a form of defense called non-host resistance is responsible for conferring immunity to a plant species against all races of a potential pathogen (Senthil-Kumar and Mysore, 2013). Non-host resistance is classified into type I and type II resistance (Mysore and Ryu, 2004). Type I resistance does not produce any visible symptom but type II resistance shows HR- cell death as shown in $R$-gene mediated resistance (SenthilKumar and Mysore, 2013; see Supplementary Table S1 for terminologies).

These plant defense responses can be systematically categorized in two tiers (Jones and Dangl, 2006) namely, pathogen-associated molecular patterns (PAMPs) triggered immunity (PTI) and effector triggered immunity (ETI). The first tier of immunity, PTI involves perception of pathogen due to PAMPs by plant receptors called pattern recognition receptors (PRRs; Bigeard et al., 2015). This recognition leads to defense response in plant. Some pathogens surpass this tier and are not recognized by plant. Such pathogens release effectors in to the cell to suppress plant defense and acquire nutrition. Then the plant executes second tier of immunity, ETI by which plant recognizes effectors of pathogen by some resistance proteins (R-protein) and prevent pathogen infection (Jones and Dangl, 2006; see Supplementary Table S1 for terminologies). In the past a number of studies had been carried out to unravel the complex resistance mechanisms and susceptibility contributing factors in plants. Recent studies proposed a new line of thinking to explain a part of defense response through proline and 1-pyrroline-5-carboxylic acid (P5C) metabolism.

Proline is a multi-functional imino acid which confers tolerance to plants against abiotic stresses (Hare and Cress, 1997; Lehmann et al., 2010; Szabados and Savoure, 2010) and has been correlated to plant defense against pathogens (Fabro et al., 2004; Cecchini et al., 2011; Senthil-Kumar and Mysore, 2012). Plant accumulates proline by increasing its synthesis and reducing catabolism under abiotic stresses (Kishor et al., 2005; Verbruggen and Hermans, 2008; Supplementary File S1). Proline content was also increased during plant defense against pathogen in Arabidopsis thaliana (Fabro et al., 2004; Verslues and Sharma, 2010). However, proline per se has not been shown to play a role in defense against pathogen infection. Recent studies have shown that proline catabolism is enhanced during early stages of plant defense against invading pathogens (Cecchini et al., 2011). Based on the evidences from recent studies (Hellmann et al., 2000; Hu et al., 2007; Nishimura et al., 2012; Lee et al., 2013), we speculate that $\mathrm{P} 5 \mathrm{C}$, an intermediate imino acid in proline metabolism, plays important role in plant defense. So far, the role of $\mathrm{P} 5 \mathrm{C}$ in plant defense against pathogens is not compiled and discussed in the literature. This review focuses on the role of P5C and its metabolism in plant-pathogen relations and attempts to infuse new thoughts in attributing relevance of P5C metabolism in plants under pathogen infection.

\section{P5C and Its Metabolism}

P5C, an N-substituted imino acid containing imino and carboxyl functional groups (IUPAC, 1997), is an intermediate not only in proline biosynthesis but also in its catabolism (Figure 1; Supplementary Table S2). P5C is synthesized from glutamate by pyrroline-5-carboxylate synthase (P5CS; Hu et al., 1992) and then converted to proline by pyrroline-5-carboxylate reductase (P5CR; Szoke et al., 1992; Hare and Cress, 1997) in cytosol and plastids. Proline is transported into mitochondria by membrane located transporters for its catabolism. Proline dehydrogenase (ProDH) catalyzes conversion of proline to $\mathrm{P} 5 \mathrm{C}$, which is then converted to glutamate by pyrroline-5-carboxylate dehydrogenase (P5CDH) in mitochondria (Elthon and Stewart, 1981; Hare and Cress, 1997). In addition to proline catabolism by ProDH (Boggess et al., 1978; Kiyosue et al., 1996), catabolism of arginine to ornithine by arginase (ARG; Goldraij and Polacco, 2000) and later transamination of ornithine by delta-ornithine amino transferase ( $\delta$ OAT) also synthesizes P5C (Delauney et al., 1993; Roosens et al., 1998; Sekhar et al., 2007; Funck et al., 2008; Stránská et al, 2008; Supplementary Table S2; Figure 1). P5C remains in rapid equilibrium with glutamate semi-aldehyde (GSA; Vogel and Davis, 1952). This equilibrium is $\mathrm{pH}$ dependent and $\mathrm{P} 5 \mathrm{C}$ form is favored over GSA at physiological pH of around 7.0 (Lewis et al., 1993; Bearne and Wolfenden, 1995).

\section{Mitochondrial ROS Accumulation and Cell Death}

In plant cells, mitochondria is one of the major sites for the production of reactive oxygen species (ROS) namely superoxide radicals and hydroxyl radicals (Lam et al., 2001; Moller, 2001; Bailey-Serres and Mittler, 2006; Sharma et al., 2012). Electrons are derived from metabolic reducing equivalents [NAD (P) $\mathrm{H}_{2}$ and FAD $(\mathrm{P}) \mathrm{H}_{2}$ ] and fed into mitochondrial electron transport chain (mETC) through complex I (NADH-ubiquinone oxidoreductase) and/or II (succinate-coenzyme Q reductase) and later transferred to oxygen via terminal oxidase to produce water (Moller, 2001; Arora et al., 2002; Turrens, 2003; Murphy, 2009; Schertl and Braun, 2014). But these carriers can also pass electron directly to oxygen to form superoxide which later produces other forms of ROS and this contributes to cell death (Fredlund, 1996; Lam et al., 2001). A localized cell death known as HR that prevents progression of pathogen infection is an important component of plant defense (Alvarez et al., 1998; Lam et al., 2001). Proline catabolism pathway mediated by ProDH enzyme in mitochondria is one of the sources of mitochondrial ROS during avirulent pathogen infection in A. thaliana (Cecchini 


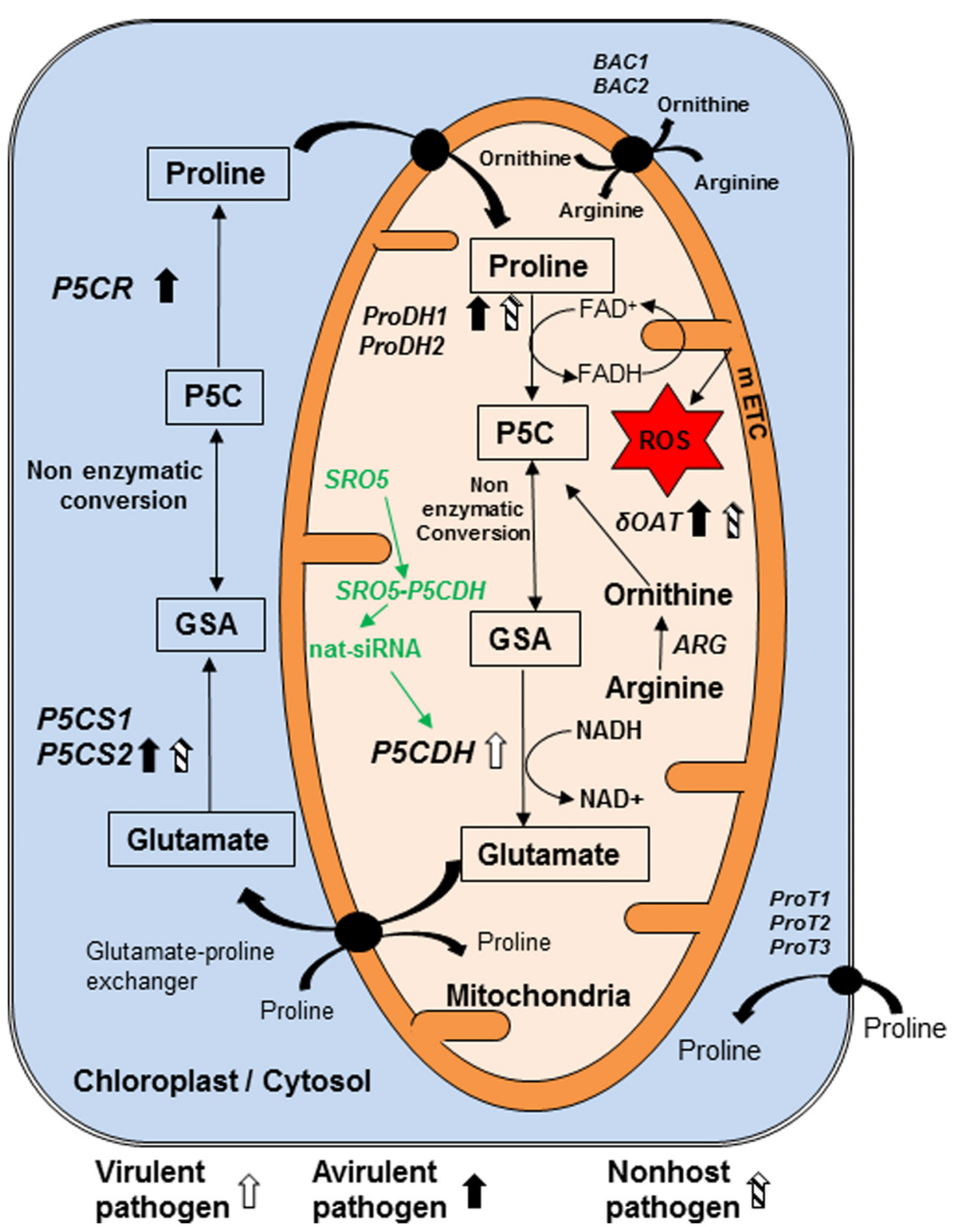

FIGURE 1| Model showing genes and pathways possibly involved in synthesis and catabolism of P5C in plant cell and their regulation in response to pathogen infection. Pyrroline 5-carboxylate (P5C) is the intermediate product of both biosynthesis and catabolism of proline. It is synthesized in mitochondria during catabolism of proline by enzyme proline dehydrogenase (ProDH1/2). We speculate that like their counterparts from bacteria and yeast, this enzyme reduces FAD+ to $\mathrm{FADH}$ and increases electron flow in mitochondrial electron transport chain (mETC). Arginine is converted into ornithine by arginase (ARG) enzyme. Another enzyme delta-ornithine amino transferase (SOAT) convert ornithine to $\mathrm{P} 5 \mathrm{C}$ in mitochondria. $\mathrm{P} 5 \mathrm{C}$ is catabolized by pyrroline 5-carboxylate dehydrogenase $(\mathrm{P} 5 \mathrm{CDH})$ in mitochondria into glutamate. In addition, P5C is synthesized in cytosol and chloroplast, from glutamate by pyrroline 5-carboxylate synthase 1 and 2 (P5CS1, P5CS2) and converted to proline by pyrroline 5-carboxylate reductase (P5CR). P5C and glutamate semi aldehyde (GSA) are non-enzymatically inter-convertible forms. Virulent pathogen infection in plants increases transcript accumulation of ProDH1. Avirulent pathogen infection increases transcript accumulation of P5CS2 and ProDH1. Non-host pathogen infection increases transcript accumulation of $\delta O A T$ as well as PrODH1 and P5CS2. Three transporters located on plasma membrane are ProT1, ProT2 and ProT3. Arginine is imported into mitochondria directly and/or in exchange of ornithine by basic amino acid career (BAC1) and BAC2. $P 5 C D H$ gene is down regulated post transcriptionally by natural siRNAs from similar to RCD one-5 (SRO5) genes. No direct evidence available for this pathway shown in green color during plant-pathogen interaction and this information is speculated based on evidence available under salt stress. Compounds shown in rectangle are important component of metabolism of P5C and line arrows indicates the direction of synthesis. Curved arrows show the transport of compounds. Transporters shown in circle are present on membrane. Block thick arrows show upregulation of genes, in which white arrow indicates virulent pathogen, dark arrows represent avirulent pathogen and striped arrows indicate non-host pathogen. ROS, reactive oxygen species. This model is predominantly based on information from Arabidopsis thaliana literature (Ayliffe et al., 2002; Borsani et al., 2005; Miller et al., 2009; Verslues and Sharma, 2010; Cecchini et al., 2011; Senthil-Kumar and Mysore, 2012). 
et al., 2011) and the ROS produced at the infection site leads to oxidative burst thereby initiates HR (Lamb and Dixon, 1997).

There are two schools of thoughts regarding ROS production by $\mathrm{P} 5 \mathrm{C}$ metabolism in mitochondria. First one emphasizes the involvement of proline-P5C cycle (Miller et al., 2009) and the extended pathway involving proline-P5C-glutamate in ROS generation. According to proline-P5C cycle, P5C synthesized due to proline catabolism in mitochondria is likely to be transported through an unknown transporter into the cytosol where P5CR enzyme converts it into proline. The proline thus formed is transported back to mitochondria where it consistently acts as a substrate for ProDH enzyme (Miller et al., 2009). In some cases, pathogen infection increased the ProDH transcript level and enhanced proline catabolism triggered proline-P5C-proline cycle between mitochondria and cytosol (Cecchini et al., 2011; Monteoliva et al., 2014). This cycle can increase the transfer of reducing equivalents to mitochondria and alter $\mathrm{NADP}^{+} / \mathrm{NADPH}$ ratio in the cytosol (Rejeb et al., 2014). This condition potentially impacts the redox sensitive pathways such as defense-associated oxidative pentose phosphate pathway and eventually produces ROS (Hare and Cress, 1997). ProDH is located in inner membrane of mitochondria toward matrix where it oxidizes proline into $\mathrm{P} 5 \mathrm{C}$ and increases electron flow leading to the production of another batch of ROS (Tanner, 2008; Wanduragala et al., 2010; Cecchini et al., 2011; Supplementary Figure S2). In Trypanosoma cruzi parasite, TcProDH mutation resulted in resistance to oxidative imbalances and ProDH enzyme activity was implicated in ROS production in this study (Paes et al., 2013). However, occurrence of proline-P5C cycle is not conclusively proven. Presence of a $\mathrm{P} 5 \mathrm{C}$ transporter at the mitochondrial membrane has only been suggested (Miller et al., 2009) but not yet identified. Similarly the role of genes encoding the mitochondrial transporters that are needed for proline cycle are not well studied. Apart from proline-P5C cycle described above, cyclic metabolisms of proline also occur by other pathways. For example, the extended pathway involving glutamate formation in mitochondria and its cycling back to cytosol for proline synthesis can also be a source of ROS production. However, till date this pathway has not been studied under plant-pathogen interaction.

The second school of thought suggests the role for $\mathrm{P} 5 \mathrm{C}$ per se in ROS production. Exogenous application of P5C led to apoptosis in human tumor cells through oxidative burst (Maxwell and Davis, 2000). In yeast, overexpression of proline utilization (PUT1) gene, which mediates the conversion of proline to $\mathrm{P} 5 \mathrm{C}$, induced cell death (Chen et al., 2006). The put2 (PUT2 gene mediates the conversion of $\mathrm{P} 5 \mathrm{C}$ to glutamate) mutant grown in proline supplemented minimal medium inhibited yeast growth due to higher ROS production (Deuschle et al., 2001). Similarly, put2 mutant of a fungal pathogen (Cryptococcus neoformans) generated high amount of mitochondrial superoxide radicals and showed cell death in proline supplemented medium (Lee et al., 2013). In addition, overexpression of ProDH induced P5C dependent mitochondria-mediated apoptosis in colorectal cancer cells ( $\mathrm{Hu}$ et al., 2007). Proline-induced cell death in yeast was also shown to be due to increased P5C levels in mitochondria (Nomura and Takagi, 2004). Nishimura et al.
(2012) reported that accumulation of P5C is responsible for mitochondrial ROS production and hence cell death in yeast. Similarly, A. thaliana $p 5 c d h$ mutant, which cannot catabolize P5C, and ProDH overexpression lines accumulated P5C in mitochondria and were hypersensitive to exogenous application of proline, ornithine, and arginine (Deuschle et al., 2004). The enhanced catabolism of proline due to increased expression of AtProDH in the $p 5 c d h$ mutant plants led to P5C accumulation with concomitant cell death (Deuschle et al., 2004). Also, AtP5CDH overexpressed plants did not show cell death but wildtype $A$. thaliana plants grown on MS medium supplemented with P5C showed hypersensitivity (Miller et al., 2009). These evidences suggest the role for P5C per se in ROS generation and eventually cell death (Supplementary Figure S2). P5C spray on wild-type $A$. thaliana leaves resulted in HR like cell death with concomitant callose accumulation (Deuschle et al., 2004). Similarly, proline hypersensitivity in wild-type A. thaliana leaves manifested as necrosis and browning symptoms were attributed to increased cellular P5C (Hellmann et al., 2000). Taken together, these studies indicate that proline-P5C cycle and/or P5C per se can contribute to HR to confine pathogen growth in plants.

\section{Role of P5C Metabolism During Plant-Avirulent Pathogen Interactions}

HR occurs during interaction of plant with either a host pathogen carrying avirulent (Avr) gene, for which plant contains corresponding $R$ gene, or a non-host pathogen (Ausubel et al., 1995; Boyes et al., 1998; Mysore and Ryu, 2004; Senthil-Kumar and Mysore, 2013). Role of P5C metabolism under both type of resistance are discussed in this section. Pseudomonas syringae pv tomato DC3000 (causal agent of bacterial spec disease in tomato) carrying AvrRpm1 or AvrRpt2 gene produces AvrRPM1 or AvrRPT2 effector protein, respectively. These are recognized by resistance to $P$. syringae maculicola 1 (RPM1) or resistance to P. syringae 2 (RPS2) protein, respectively, in host plant through RPM interacting protein (RIN4) (Mackey et al., 2002; Axtell and Staskawicz, 2003). This recognition leads to changes in prolineP5C metabolism (Cecchini et al., 2011; Monteoliva et al., 2014) and up regulation of various defense genes and ROS production culminating in HR-cell death (Torres et al., 2006). External proline application, that can provoke $\mathrm{P} 5 \mathrm{C}$ accumulation induces pathogenesis related $1(P R 1)$ gene expression in $A$. thaliana (Chen et al., 2011). These recent studies highlight the role of P5C and its metabolism in inducing defense responses, including HR during plant-pathogen interactions.

Fabro et al. (2004) reported the accumulation of AtP5CS2 transcripts and increased proline content prior to $\mathrm{HR}$ in A. thaliana plants infected with avirulent pathogens ( $P$. syringae pv tomato DC3000 AvrRpt2 and P. syringae pv tomato DC3000 AvrRpm1). Cecchini et al. (2011) reported upregulation of AtProDH1 and AtProDH2 genes in A. thaliana at the inoculation site of avirulent pathogen (P. syringae pv tomato DC3000 AvrRpm1). In addition, avirulent pathogen inoculation led to increased ProDH protein content and enzyme activity and was 
suggested to be responsible for oxidative burst and HR (Cecchini et al., 2011). Consistently, AtProDH silenced A. thaliana plants inoculated with avirulent pathogen compromised ROS production (Cecchini et al., 2011). AtProDH gene transcript expression was modulated by exogenous application of salicylic acid (SA, Cecchini et al., 2011). Authors in this study proposed that SA-mediated signaling plays a role in the induction of AtProDH1, but not AtProDH2, expression likely through nonexpressor of $P R$ genes 1 (NPR1) and SA induction-deficient 2 (SID2) during early stages of avirulent pathogen infection in A. thaliana. Proline was shown to induce SA via non-responsive to disease resistance 1 (NDR1)-dependent pathway (Vlot et al., 2009; Chen et al., 2011). In green bean (Phaseolus vulgaris), Rhizoctonia solani (causal agent of seed rot and damping off in bean) infection induced proline accumulation in SA-dependent manner (Ayoubi and Soleimani, 2014). Taken together, these studies implicate a role for AtP5CS2 and AtProDH1 genes in regulating the proline and $\mathrm{P} 5 \mathrm{C}$ under Avr-R interaction. The defense responses involving these genes are likely part of ETI and involve SA.

Further, AtP5CDH gene transcription was downregulated in $A$. thaliana upon inoculation with $P$. syringae pv. tomato DC3000 AvrRpm1. Consistently in $p 5 c d h$ mutant of A. thaliana, slight increase in $\mathrm{P} 5 \mathrm{C}$ level was observed in the $\mathrm{HR}$ region upon inoculation by P. syringae pv. tomato DC3000 AvrRpm1 (Monteoliva et al., 2014). This scenario facilitate decrease in degradation of $\mathrm{P} 5 \mathrm{C}$ and hence results in high accumulation of P5C (Supplementary Figures S1 and S2). Notably, slight increase in concentration of $\mathrm{P} 5 \mathrm{C}$ due to induction of $\mathrm{P} 5 \mathrm{C}$ biosynthesis by avirulent pathogen inoculation had been shown to provoke HR (Hellmann et al., 2000; Nishimura et al., 2012). It is likely that $A t P 5 C D H$-mediated P5C regulation is also a part of ETI.

Similar to avirulent pathogens, non-host pathogen infection also involves $\mathrm{HR}$ and other defenses likely mediated by P5C metabolism (Senthil-Kumar and Mysore, 2012, 2013). Recently, virus-induced gene silencing (VIGS)-based forward genetics screen identified the role for ProDH1 and $\delta O A T$ in nonhost resistance (Senthil-Kumar and Mysore, 2012). Silencing of NbSOAT and NbProDH1 genes compromised non-host resistance against $P$. syringae pv. tomato $\mathrm{T} 1$ in Nicotiana benthamiana. Further, A. thaliana $\delta$ oat and $p 5 c d h$ mutant plants lacked oxidative burst and compromised non-host resistance against P. syringae pv. tabaci (Senthil-Kumar and Mysore, 2012). These mutant plants grown in Murashige and Skoog (MS) medium externally supplemented with P5C showed higher ROS content as compared to those grown in non-P5C medium. Taken together, this study suggested the possible involvement of P5C synthesis step in non-host resistance, possibly as a part of both PTI and ETI.

\section{Role of P5C Metabolism During Plant-Virulent Pathogen Interaction}

Flax (Linum usitatissimum) plant infected with the Melampsora lini (causal agent of flax rust), an obligate biotrophic fungal pathogen, induced the FIS1 (flax inducible sequence 1) gene at the pathogen infection site (Ayliffe et al., 2002). Interestingly, FIS1 gene showed 73\% nucleotide homology with the A. thaliana AtP5CDH gene (Mitchell et al., 2006). Similarly, $N$. benthamiana plants infected with virulent pathogen (P. syringae pv. tabaci) showed upregulation of $\mathrm{NbP5CDH}$ gene expression (Senthil-Kumar and Mysore, 2012). It is possible that virulent pathogens enhance the catabolism of $\mathrm{P} 5 \mathrm{C}$ by upregulating AtP5CDH-mediated step (Figure 2; Supplementary Figure $\mathrm{S} 1$ ). In $A$. thaliana, infection by virulent pathogen (P. syringae pv. tomato DC3000) did not lead to upregulation of ProDH gene (Cecchini et al., 2011). Similarly, N. benthanmiana plants also did not show upregulation of $\mathrm{NbProDH} 1$ and $\mathrm{NbProDH} 2$ gene expression at $\mathrm{P}$. syringae pv. tabaci infection site (Senthil-Kumar and Mysore, 2012). This indicates that plants attempt to maintain proline levels in the cells undergoing disease induced cell death. This is consistent with previous studies that indicated the role of proline in reducing cell death in yeast (Chen et al., 2006), fungus (Chen and Dickman, 2005), and plants (Banu et al., 2009). Proline provides protection against $\mathrm{H}_{2} \mathrm{O}_{2}$-induced cell death and apoptosis in mammalian cell culture (Krishnan et al., 2008). Taken together, these studies demonstrated that proline, not $\mathrm{P} 5 \mathrm{C}$, is likely to play a role in regulating diseaseinduced cell death (Figure 2). In addition to bacterial and fungal pathogens, virus infection was shown to influence proline-P5C metabolism. For example, rice plants infected with Brome mosaic virus (BMV) or Rice tungro virus led to proline accumulation (Mohanty and Sridhar, 1982; Xu et al., 2008). However, the positive role of proline in cell death regulation is not examined in these studies.

\section{Coordinated Regulation of Proline-P5C Metabolism During Plant Defense}

High proline accumulation in non-stressed plants has been shown to negatively impact plant growth and also cause cell death (Hellmann et al., 2000; Chen et al., 2011). However, when these plants are subjected to stress the toxicity due to proline accumulation is reduced. The proline content increases upon pathogen infection (Fabro et al., 2004), but exogenous proline application to non-stressed plants cause increase in its catabolism (Verslues and Sharma, 2010). Proline itself at low concentrations is known to reduce the cell death, but $\mathrm{P} 5 \mathrm{C}$ metabolism provokes cell death (Deuschle et al., 2004; Banu et al., 2009). These evidences indicate the complex but finetuned regulation of proline-P5C metabolism during pathogen infection (Figure 2). It is possible that in virulent pathogen infected cells, proline acts as a positive regulator of cell death as part of basal immune response to decrease the severity of disease development. It is also possible that the un-infected cells surrounding the HR developing cells upon inoculation with avirulent pathogen increase proline levels to prevent runaway cell death (Senthil-Kumar and Mysore, 2012). In contrary, either proline-P5C cycle or P5C accumulation per se triggers the defense cell death (HR) upon avirulent pathogen infection. In order to bring a fine-tuned regulation of proline-P5C pathway 


\section{Compatible interaction}

\section{Incompatible interaction}

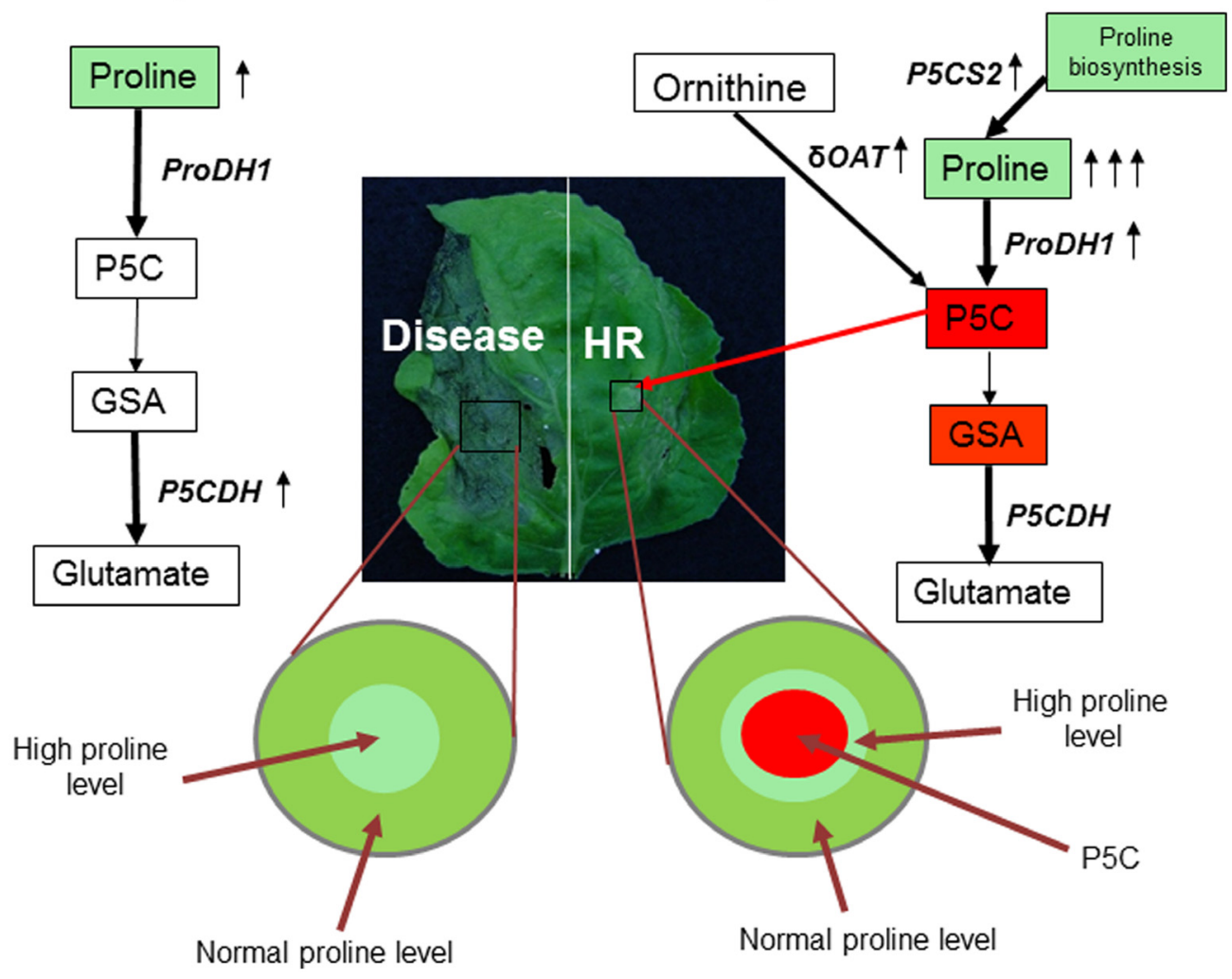

FIGURE 2 | Model for coordinated regulation of proline-P5C metabolism under pathogen infection. Virulent pathogen (example as shown here for Pseudomonas syringae pv. tabaci in Nicotiana benthamiana) infection leads to disease on host plant (left). However, non-host (example as shown here for $P$. syringae pv. tomato $T 1$ in $N$. benthamiana) [and avirulent, not shown in the picture] pathogen infection on plant leads to defense reaction, including HR at infection site (right). Pathways leading to P5C biosynthesis and catabolism in mitochondria are highlighted. Plants increase P5C content or ProDH-mediated step and produce ROS to defend against pathogen by upregulating ProDH and $\delta O A T$ gene transcription. In addition, mild increase in proline in the tissues surrounding the HR cell death prevents runaway HR to non-infected cells. However, virulent pathogens decrease P5C levels in mitochondria by upregulating P5CDH-mediated step. Also proline content moderately increases in the infected cells possibly to decrease the rate of disease progression as a part of basal defense response. Proline is known to prevent or delay the cell death. During this regulation, proline synthesis can also play a role in controlling proline levels (shown under resistant reaction). Red rectangle box depicts pro cell death compound; green rectangle depicts compound that delays cell death; arrow depicts upregulation of transcripts. the plant must regulate the genes involved in defense through a systematic network. We speculate that this network may involve a tight regulation at the promoters. Yet another possibility of post transcriptional regulation could involve micro RNAs (miRNAs). In potato, miRNAs targeting proline metabolism genes were predicted and their role in regulation was verified (Yang et al., 2013). We speculate that proline metabolism genes can be regulated by microRNAs under pathogen attack as well.

Additionally, expression of proline metabolism genes is differentially regulated by drought, salinity, and abscisic acid suggesting that these genes play a specific role in the control of proline synthesis (Supplementary File S1). This indicates the coordinated regulation of proline-P5C metabolism under abiotic stress as well. Considering literature information available on their role under abiotic stresses, we comprehensively illustrated this information together with the regulation under plant-pathogen interaction. Both proline and P5C content under various abiotic stresses changes and regulation of genes involved in different steps of proline catabolism can be correlated to their levels under pathogen stress.

\section{Concluding Remarks}

P5C plays a role in HR against invading pathogen and its levels or metabolism could possibly be manipulated by virulent pathogen. To know the importance of its metabolism in plant defense, it is necessary to understand the regulation of genes involved in its pathway at transcriptional and post transcriptional level. Bioinformatic analysis and experimental validation of promoter region of all proline-P5C metabolism genes will be useful for understanding the regulatory components. In addition, studies to understand post transcriptional modifications, 
cofactor binding, protein interactors and activity of all proteins involved in the pathway during plant-pathogen interaction are required.

It is still not clear whether the changes in proline-P5C metabolism are primarily regulated by ETI or PTI or both. Systematic studies that will exclusively use PAMPs, effectors and specific triggers for innate immunity is needed to delineate the defense pathway that provoke changes in P5C metabolism. In addition to this, the downstream defense pathway activated by $\mathrm{P} 5 \mathrm{C}$ that culminates in $\mathrm{HR}$ or other defense responses is also not well understood. Studies using externally supplied purified $\mathrm{P} 5 \mathrm{C}$ and genetically manipulated plants with altered P5C levels under a specific defense inducer can provide useful information. Non-host plant-pathogen interaction can be used as model to study both defense involving $\mathrm{HR}$ and others. This is because both type-I (HR not involved) and type-II (involving HR) defenses can be tested using the same plant species.

More studies are needed to understand whether P5C content in mitochondria, suppression of its catabolism or its transport plays a role in plant defense. The contribution of cytosolic P5C in pathogen induced plant defense is also not yet studied. In order to understand whether the level of P5C plays any role in triggering $\mathrm{HR}$, it is necessary to accurately estimate the P5C content. However, currently available methods do not provide organelle specific accurate estimation of this compound. For

\section{References}

Alvarez, M. E., Pennell, R. I., Meijer, P. J., Ishikawa, A., Dixon, R. A., and Lamb, C. (1998). Reactive oxygen intermediates mediate a systemic signal network in the establishment of plant immunity. Cell 92, 773-784. doi: 10.1016/S00928674(00)81405-1

Arora, A., Sairam, R. K., and Srivastava, G. C. (2002). Oxidative stress and antioxidative system in plants. Curr. Sci. 82, 1227-1238.

Ausubel, F. M., Katagiri, F., Mindrinos, M., and Glazebrook, J. (1995). Use of Arabidopsis thaliana defense-related mutants to dissect the plant response to pathogens. Proc. Natl. Acad. Sci. U.S.A. 92, 4189-4196. doi: 10.1073/pnas.92.10.4189

Axtell, M. J., and Staskawicz, B. J. (2003). Initiation of RPS2-specified disease resistance in Arabidopsis is coupled to the AvrRpt2-directed elimination of RIN4. Cell 112, 369-377. doi: 10.1016/S0092-8674(03) 00036-9

Ayliffe, M. A., Roberts, J. K., Mitchell, H. J., Zhang, R., Lawrence, G. J., Ellis, J. G., et al. (2002). A plant gene up-regulated at rust infection sites. Plant Physiol. 129, 169-180. doi: 10.1104/pp.010940

Ayoubi, N., and Soleimani, M. J. (2014). Possible effects of pathogen inoculation and salicylic acid pre-treatment on the biochemical changes and proline accumulation in green bean. Arch. Phytopathol. Plant Prot. 48, 212-222. doi: $10.1080 / 03235408.2014 .884826$

Bailey-Serres, J., and Mittler, R. (2006). The roles of reactive oxygen species in plant cells. Plant Physiol. 141, 311. doi: 10.1104/pp.104.900191

Banu, M. N. A., Hoque, M. A., Watanabe-Sugimoto, M., Matsuoka, K., Nakamura, Y., Shimoishi, Y., et al. (2009). Proline and glycinebetaine induce antioxidant defense gene expression and suppress cell death in cultured tobacco cells under salt stress. J. Plant Physiol. 166, 146-156. doi: 10.1016/j.jplph.2008.03.002

Bearne, S. L., and Wolfenden, R. (1995). Glutamate gamma-semialdehyde as a natural transition state analogue inhibitor of Escherichia coli glucosamine6-phosphate synthase. Biochemistry 34, 11515-11520. doi: 10.1021/bi00 $036 \mathrm{a} 026$ example, colorimetric method of $\mathrm{P} 5 \mathrm{C}$ estimation by $\mathrm{O}$-aminobenzaldehyde assay is not sensitive enough to estimate P5C content in vivo. Hence, development of suitable P5C estimation method is important.

Since proline is well known to play a positive role during drought stress and also during stress recovery, it will be interesting to study the plant's interaction with virulent and avirulent pathogens under these two conditions. Further, studies on proline pathway gene regulation under the combined drought and pathogen infection will be useful.

\section{Acknowledgments}

This work was supported by the core funding to MS-K's lab from National Institute of Plant Genome Research, New Delhi. AQ thanks the University Grant Commission (UGC), New Delhi for a junior research fellowship award (Ref. No. 23/12/2012(ii) EUV). Authors also thank Mr. Mehanathan Muthamilarasan and Dr. Prachi Pandey for critical reading of the manuscript.

\section{Supplementary Material}

The Supplementary Material for this article can be found online at: http://journal.frontiersin.org/article/10.3389/fpls.2015.00503

Bigeard, J., Colcombet, J., and Hirt, H. (2015). Signaling mechanisms in pattern-triggered immunity (PTI). Mol. Plant 8, 521-539. doi: 10.1016/j.molp.2014.12.022

Boggess, S. F., Koeppe, D. E., and Stewart, C. R. (1978). Oxidation of proline by plant mitochondria. Plant Physiol. 62, 22-25. doi: 10.1104/pp.62.1.22

Borsani, O., Zhu, J., Verslues, P. E., Sunkar, R., and Zhu, J.-K. (2005). Endogenous siRNAs derived from a pair of natural cis-antisense transcripts regulate salt tolerance in Arabidopsis. Cell 123, 1279-1291. doi: 10.1016/j.cell.2005. 11.035

Boyes, D. C., Nam, J., and Dangl, J. L. (1998). The Arabidopsis thaliana RPM1 disease resistance gene product is a peripheral plasma membrane protein that is degraded coincident with the hypersensitive response. Proc. Natl. Acad. Sci. U.S.A. 95, 15849-15854. doi: 10.1073/pnas.95.26. 15849

Cecchini, N. M., Monteoliva, M. I., and Alvarez, M. E. (2011). Proline dehydrogenase contributes to pathogen defense in Arabidopsis. Plant Physiol. 155, 1947-1959. doi: 10.1104/pp.110.167163

Chen, C., and Dickman, M. B. (2005). Proline suppresses apoptosis in the fungal pathogen Colletotrichum trifolii. Proc. Natl. Acad. Sci. U.S.A. 102, 3459-3464. doi: 10.1073/pnas.0407960102

Chen, C., Wanduragala, S., Becker, D. F., and Dickman, M. B. (2006). Tomato QMlike protein protects Saccharomyces cerevisiae cells against oxidative stress by regulating intracellular proline levels. Appl. Environ. Microbiol. 72, 4001-4006. doi: 10.1128/AEM.02428-05

Chen, J., Zhang, Y., Wang, C., Lü, W., Jin, J. B., and Hua, X. (2011). Proline induces calcium-mediated oxidative burst and salicylic acid signaling. Amino Acids 40, 1473-1484. doi: 10.1007/s00726-010-0757-2

Delauney, A., Hu, C., Kishor, P. K., and Verma, D. (1993). Cloning of ornithine d-aminotransferase cDNA by trans-complementation in Escherichia coli and regulation of proline biosynthesis. J. Biol. Chem. 268, 1867318678.

Deuschle, K., Funck, D., Forlani, G., Stransky, H., Biehl, A., Leister, D., et al. (2004). The role of $\Delta 1$-pyrroline-5-carboxylate dehydrogenase in proline degradation. Plant Cell 16, 3413-3425. doi: 10.1105/tpc.104.023622 
Deuschle, K., Funck, D., Hellmann, H., Daschner, K., Binder, S., and Frommer, W. B. (2001). A nuclear gene encoding mitochondrial $\Delta$ 1-pyrroline-5carboxylate dehydrogenase and its potential role in protection from proline toxicity. Plant J. 27, 345-355. doi: 10.1046/j.1365-313X.2001.01101.x

Elthon, T. E., and Stewart, C. R. (1981). Submitochondrial location and electron transport characteristics of enzymes involved in proline oxidation. Plant Physiol. 67, 780-784. doi: 10.1104/pp.67.4.780

Fabro, G., Kovacs, I., Pavet, V., Szabados, L., and Alvarez, M. E. (2004). Proline accumulation and AtP5CS2 gene activation are induced by plant-pathogen incompatible interactions in Arabidopsis. Mol. Plant Microbe Interact. 17, 343-350. doi: 10.1094/MPMI.2004.17.4.343

Fredlund, K. M. (1996). NAD $(P) H$ Dehydrogenases in Plant Mitochondria. Ph.D. Thesis, Lund University, Lund.

Funck, D., Stadelhofer, B., and Koch, W. (2008). Ornithine- $\delta$-aminotransferase is essential for arginine catabolism but not for proline biosynthesis. BMC Plant Biol. 8:40. doi: 10.1186/1471-2229-8-40

Goldraij, A., and Polacco, J. C. (2000). Arginine degradation by arginine in mitochondria of soybean seedling cotyledons. Planta 4, 652-658. doi: 10.1007/s004250050056

Hare, P. D., and Cress, W. A. (1997). Metabolic implications of stressinduced proline accumulation in plants. Plant Growth Regul. 21, 79-102. doi: 10.1023/A:1005703923347

Hellmann, H., Funck, D., Rentsch, D., and Frommer, W. B. (2000). Hypersensitivity of an Arabidopsis sugar signaling mutant toward exogenous proline application. Plant Physiol. 123, 779-789. doi: 10.1104/pp.123.2.779

$\mathrm{Hu}$, C. A., Delauney, A. J., and Verma, D. P. (1992). A bifunctional enzyme ( $\Delta$ 1-pyrroline-5-carboxylate synthetase) catalyzes the first two steps in proline biosynthesis in plants. Proc. Natl. Acad. Sci. U.S.A. 89, 9354-9358. doi: 10.1073/pnas.89.19.9354

Hu, C. A. A., Donald, S. P., Yu, J., Lin, W. W., Liu, Z., Steel, G., et al. (2007). Overexpression of proline oxidase induces proline-dependent and mitochondria-mediated apoptosis. Mol. Cell. Biochem. 295, 85-92. doi: 10.1007/s11010-006-9276-6

IUPAC. (1997). “Compendium of Chemical Terminology," in The Gold Book, 2nd Edn, eds A. D. McNaught and A. Wilkinson (Oxford: Blackwell Scientific Publications).

Jones, J. D., and Dangl, J. L. (2006). The plant immune system. Nature 444, 323-329. doi: 10.1038/nature05286

Kishor, P. K., Sangam, S., Amrutha, R. N., Laxmi, P. S., Naidu, K. R., Rao, K. R. S. S., et al. (2005). Regulation of proline biosynthesis, degradation, uptake and transport in higher plants: its implications in plant growth and abiotic stress tolerance. Curr. Sci. 88, 424-438.

Kiyosue, T., Yoshiba, Y., Yamaguchi-Shinozaki, K., and Shinozaki, K. (1996). A nuclear gene encoding mitochondrial proline dehydrogenase, an enzyme involved in proline metabolism, is upregulated by proline but downregulated by dehydration in Arabidopsis. Plant Cell 8, 1323-1335. doi: 10.1105/tpc.8. 8.1323

Krishnan, N., Dickman, M. B., and Becker, D. F. (2008). Proline modulates the intracellular redox environment and protects mammalian cells against oxidative stress. Free Radic. Biol. Med. 44, 671-681. doi: 10.1016/j.freeradbiomed.2007.10.054

Lam, E., Kato, N., and Lawton, M. (2001). Programmed cell death, mitochondria and the plant hypersensitive response. Nature 411, 848-853. doi: $10.1038 / 35081184$

Lamb, C., and Dixon, R. A. (1997). The oxidative burst in plant disease resistance. Annu. Rev. Plant Physiol. Plant Mol. Biol. 48, 251-275. doi: 10.1146/annurev.arplant.48.1.251

Lee, I. R., Lui, E. Y., Chow, E. W., Arras, S. D., Morrow, C. A., and Fraser, J. A. (2013). Reactive oxygen species homeostasis and virulence of the fungal pathogen Cryptococcus neoformans requires an intact proline catabolism pathway. Genetics 194, 421-433. doi: 10.1534/genetics.113.150326

Lehmann, S., Funck, D., Szabados, L., and Rentsch, D. (2010). Proline metabolism and transport in plant development. Amino Acids 39, 949-962. doi: 10.1007/s00726-010-0525-3

Lewis, M. L., Rowe, C. J., Sewald, N., Sutherland, J. D., Wilson, E. J., and Wright, M. C. (1993). The effect of $\mathrm{pH}$ on the solution structure of delta-1-pyrroline-2carboxylic acid as revealed by NMR and electrospray mass spectroscopy. Bioorg. Med. Chem. Lett. 3, 1193-1196. doi: 10.1016/S0960-894X(00)80313-3
Mackey, D., Holt, B. F. III., Wiig, A., and Dangl, J. L. (2002). RIN4 interacts with Pseudomonas syringae type III effector molecules and is required for RPM1mediated resistance in Arabidopsis. Cell 108, 743-754. doi: 10.1016/S00928674(02)00661-X

Maxwell, S. A., and Davis, G. E. (2000). Differential gene expression in p53mediated apoptosis-resistant vs. apoptosis-sensitive tumor cell lines. Proc. Natl. Acad. Sci. U.S.A. 97, 13009-13014. doi: 10.1073/pnas.230445997

Miller, G., Honig, A., Stein, H., Suzuki, N., Mitler, R., and Zilberstein, A. (2009). Unraveling delta1-pyrroline-5-carboxylate-proline cycle in plants by uncoupled expression of proline oxidation enzymes. J. Biol. Chem. 289, 26482-26492. doi: 10.1074/jbc.M109.009340

Mitchell, H. J., Ayliffe, M. A., Rashid, K. Y., and Pryor, A. J. (2006). A rust-inducible gene from flax (fis1) is involved in proline catabolism. Planta 223, 213-222. doi: $10.1007 /$ s00425-005-0079-x

Mohanty, S. K., and Sridhar, R. (1982). Physiology of rice tungro virus disease: proline accumulation due to infection. Physiol. Plant. 56, 89-93. doi: 10.1111/j.1399-3054.1982.tb04904.x

Moller, I. M. (2001). Plant mitochondria and oxidative stress: electron transport, NADPH turnover, and metabolism of reactive oxygen species. Annu. Rev. Plant Physiol. Plant Mol. Biol. 52, 561-591. doi: 10.1146/annurev.arplant. 52.1.561

Monteoliva, M. I., Rizzi, Y. S., Cecchini, N. M., Hajirezaei, M. R., and Alvarez, M. E. (2014). Context of action of proline dehydrogenase (ProDH) in the hypersensitive response of Arabidopsis. BMC Plant Biol. 14:21. doi: 10.1186/1471-2229-14-21

Murphy, M. P. (2009). How mitochondria produce reactive oxygen species. Biochem. J. 417, 1-13. doi: 10.1042/BJ20081386

Mysore, K. S., and Ryu, C. M. (2004). Nonhost resistance: how much do we know? Trends Plant Sci. 9, 97-104. doi: 10.1016/j.tplants.2003.12.005

Nishimura, A., Nasano, R., and Takagi, H. (2012). The proline metabolism intermediate (delta) 1-pyrroline-5-carboxylate directly inhibits the mitochondrial respiration in budding yeast. FEBS Lett. 586, 2411-2416. doi: 10.1016/j.febslet.2012.05.056

Nomura, M., and Takagi, H. (2004). Role of the yeast acetyltransferase Mpr1 in oxidative stress: regulation of oxygen reactive species caused by a toxic proline catabolism intermediate. Proc. Natl. Acad. Sci. U.S.A. 101, 12616-12621. doi: 10.1073/pnas.0403349101

Paes, L. S., Mantilla, B. S., Zimbres, F. M., Pral, E. M. F., Melo, P. D., Tahara, E. B., et al. (2013). Proline dehydrogenase regulates redox state and respiratory metabolism in Trypanosoma cruzi. PLoS ONE 8:e69419. doi: 10.1371/journal.pone.0069419

Rejeb, K. B., Abdelly, C., and Savouré, A. (2014). How reactive oxygen species and proline face stress together. Plant Physiol. Biochem. 80, 278-284. doi: 10.1016/j.plaphy.2014.04.007

Roosens, N. H., Thu, T. T., Iskandar, H. M., and Jacobs, M. (1998). Isolation of the ornithine- $\delta$-aminotransferase cDNA and effect of salt stress on its expression in Arabidopsis thaliana. Plant Physiol. 117, 263-271. doi: 10.1104/pp.117.1.263

Schertl, P., and Braun, H. P. (2014). Respiratory electron transfer pathways in plant mitochondria. Front. Plant Sci. 5:163. doi: 10.3389/fpls.2014.00163

Sekhar, P. N., Amrutha, R. N., Sangam, S., Verma, D. P. S., and Kishor, P. K. (2007). Biochemical characterization, homology modeling and docking studies of ornithine $\delta$-aminotransferase-an important enzyme in proline biosynthesis of plants. J. Mol. Graph. Model. 26, 709-719. doi: 10.1016/j.jmgm.2007. 04.006

Senthil-Kumar, M., and Mysore, K. S. (2012). Ornithine-delta-aminotransferase and proline dehydrogenase genes play a role in non-host disease resistance by regulating pyrroline-5-carboxylate metabolism-induced hypersensitive response. Plant Cell Environ. 35, 1329-1343. doi: 10.1111/j.1365-3040.2012.02492.x

Senthil-Kumar, M., and Mysore, K. S. (2013). Nonhost resistance against bacterial pathogens: retrospectives and prospects. Annu. Rev. Phytopathol. 51, 407-427. doi: 10.1146/annurev-phyto-082712-102319

Sharma, P., Jha, A. B., Dubey, R. S., and Pessarakli, M. (2012). Reactive oxygen species, oxidative damage, and antioxidative defense mechanism in plants under stressful conditions. J. Bot. 2012, 1-26. doi: 10.1155/2012/217037

Stránská, J., Kopečný, D., Tylichová, M., Snégaroff, J., and Šebela, M. (2008). Ornithine $\delta$-aminotransferase: an enzyme implicated in salt tolerance in higher plants. Plant Signal. Behav. 3, 929-935. doi: 10.4161/psb.6771 
Szabados, L., and Savoure, A. (2010). Proline: a multifunctional amino acid. Trends Plant Sci. 15, 89-97. doi: 10.1016/j.tplants.2009.11.009

Szoke, A., Miao, G. H., Hong, Z., and Verma, D. P. (1992). Subcellular Location of delta 1-pyrroline-5-carboxylate reductase in root/nodule and leaf of soybean. Plant Physiol. 99, 1642-1649. doi: 10.1104/pp.99.4.1642

Tanner, J. J. (2008). Structural biology of proline catabolism. Amino Acids 35, 719-730. doi: 10.1007/s00726-008-0062-5

Torres, M. A., Jones, J. D., and Dangl, J. L. (2006). Reactive oxygen species signaling in response to pathogens. Plant Physiol. 141, 373-378. doi: 10.1104/pp.106.079467

Turrens, J. F. (2003). Mitochondrial formation of reactive oxygen species. J. Physiol. 552, 335-344. doi: 10.1113/jphysiol.2003.049478

Verbruggen, N., and Hermans, C. (2008). Proline accumulation in plants: a review. Amino Acids 35, 753-759. doi: 10.1007/s00726-008-0061-6

Verslues, P. E., and Sharma, S. (2010). Proline metabolism and its implications for plant-environment interaction. Arabidopsis Book 8:e0140. doi: 10.1199/ tab. 0140

Vlot, A. C., Dempsey, D. M. A., and Klessig, D. F. (2009). Salicylic acid, a multifaceted hormone to combat disease. Annu. Rev. Phytopathol. 47, 177-206. doi: 10.1146/annurev.phyto.050908.135202

Vogel, H. J., and Davis, B. D. (1952). Glutamic $\gamma$-semi aldehyde and $\Delta 1$-pyrroline5-carboxylic acid, intermediates in the biosynthesis of proline. J. Am. Chem. Soc. 74, 109-112. doi: 10.1021/ja01121a025
Wanduragala, S., Sanyal, N., Liang, X., and Becker, D. F. (2010). Purification and characterization of Putlp from Saccharomyces cerevisiae. Arch. Biochem. Biophys. 498, 136-142. doi: 10.1016/j.abb.2010.04.020

Xu, P., Chen, F., Mannas, J. P., Feldman, T., Sumner, L. W., and Roossinck, M. J. (2008). Virus infection improves drought tolerance. New Phytol. 180, 911-921. doi: 10.1111/j.1469-8137.2008.02627.x

Yang, J., Zhang, N., Ma, C., Qu, Y., Si, H., and Wang, D. (2013). Prediction and verification of microRNAs related to proline accumulation under drought stress in potato. Comput. Biol. Chem. 46, 48-54. doi: 10.1016/j.compbiolchem.2013.04.006

Conflict of Interest Statement: The Review Editor Yasuhiro Ishiga declares that, despite having collaborated and co-authored manuscripts with the authors, Mysore and Senthil-Kumar, the review process was conducted objectively. The authors declare that the research was conducted in the absence of any commercial or financial relationships that could be construed as a potential conflict of interest.

Copyright (C) 2015 Qamar, Mysore and Senthil-Kumar. This is an open-access article distributed under the terms of the Creative Commons Attribution License (CC BY). The use, distribution or reproduction in other forums is permitted, provided the original author(s) or licensor are credited and that the original publication in this journal is cited, in accordance with accepted academic practice. No use, distribution or reproduction is permitted which does not comply with these terms. 